\title{
Disseminated histoplasmosis with oral manifestations
}

\author{
Dhammika TAN, Dias DK, Perera HAS \\ Maxillofacial and Regional Cleft Center, Teaching Hospital Karapitiya, Galle, Sri Lanka.
}

\author{
Correspondence: Dr.TAN Dhammika \\ e-mail: dhammikad888@yahoo.com
}

\section{Introduction}

Histoplasmosis is fungal infection caused by dimorphic fungus Histoplasma capsulatum. The disease is known to be endemic in some parts of North and Central America (1) but it is a very rare disease in Sri Lanka. According to the severity of the signs and symptoms and organs affected; acute, chronic, disseminated and African sub types can be identified.

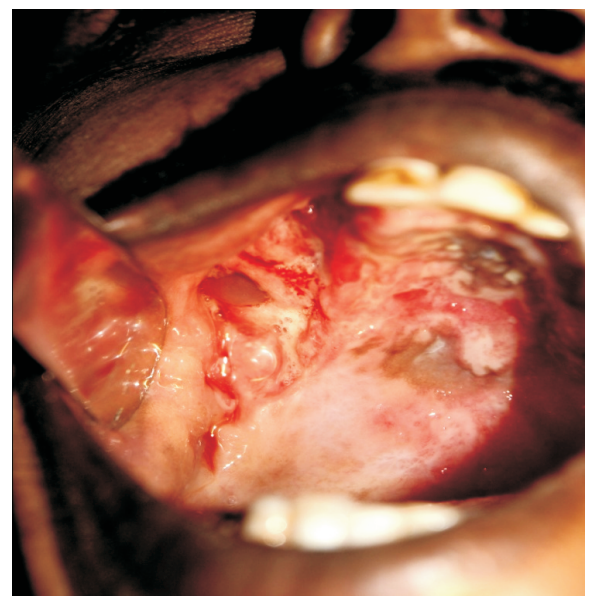

Figure 1: Ulceration in the upper buccal sulcus and the hard palate
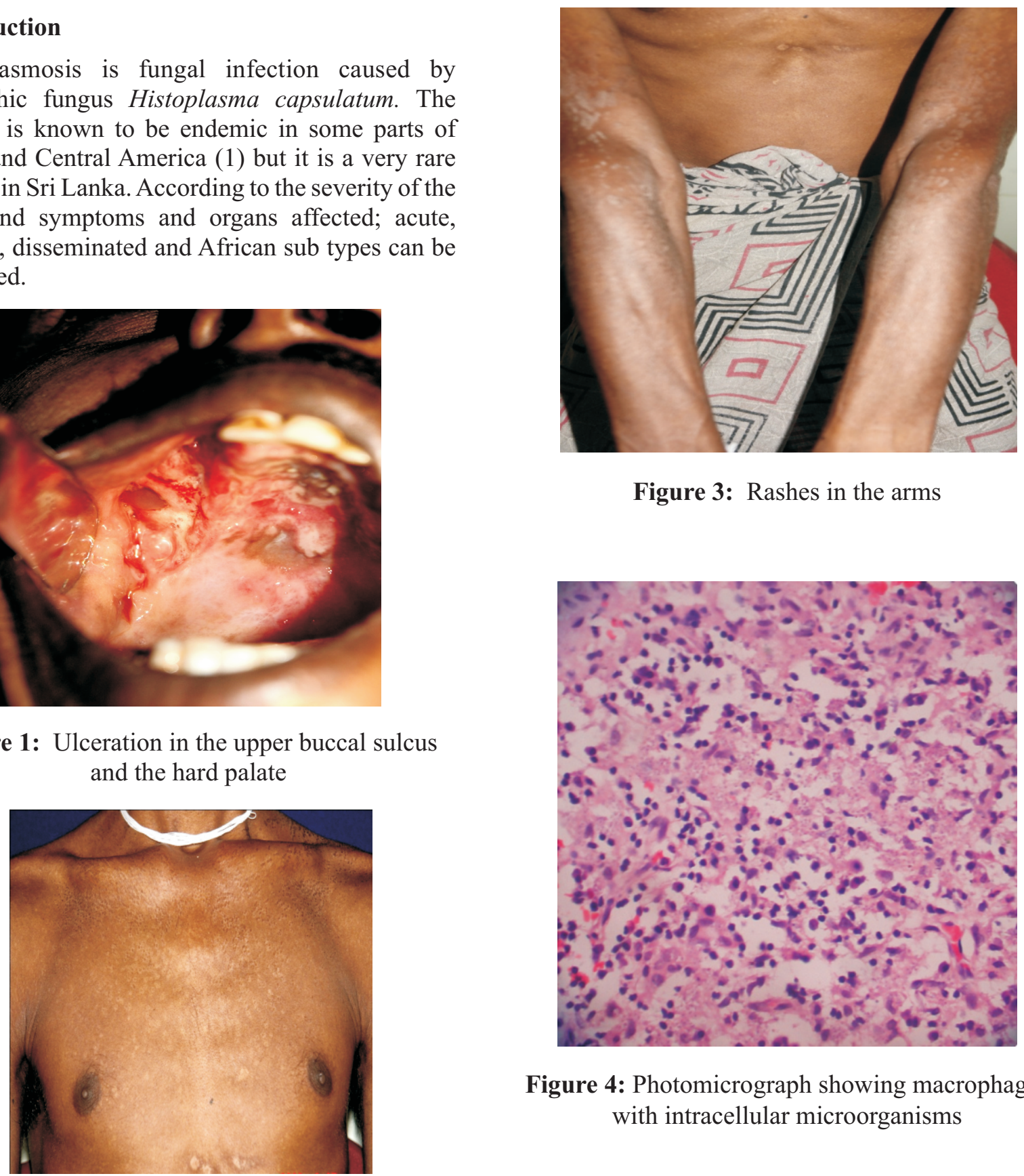

Figure 3: Rashes in the arms

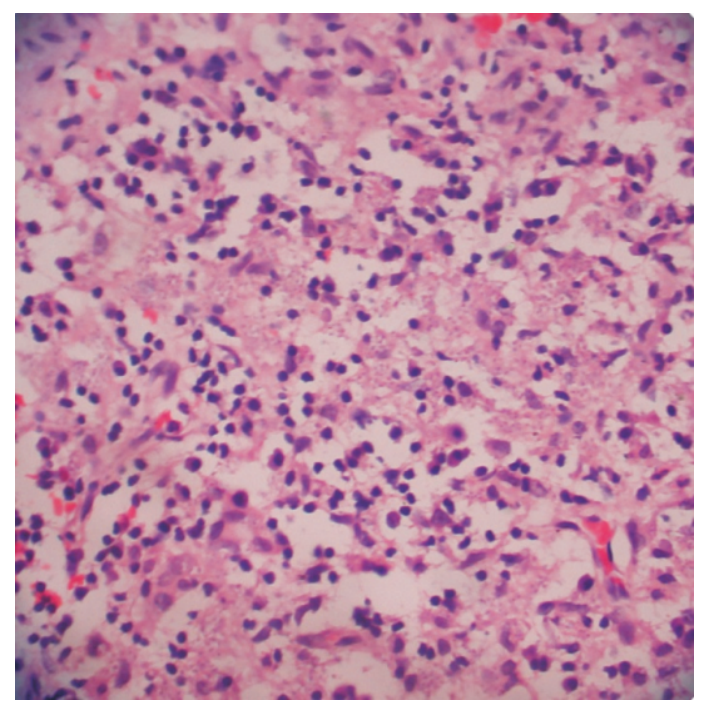

Figure 4: Photomicrograph showing macrophages with intracellular microorganisms

Figure 2: Chest ulcers and rashes 

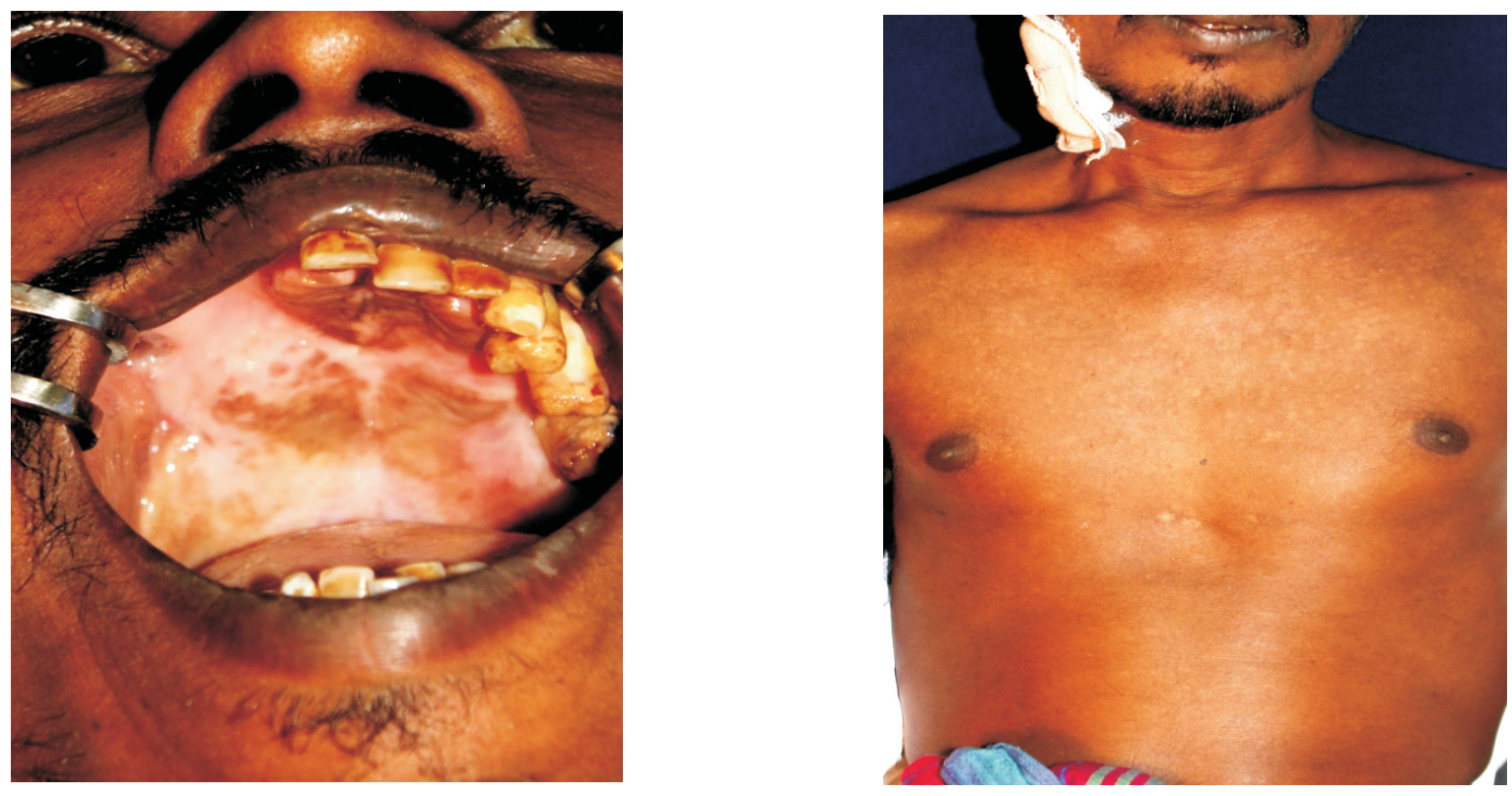

Figure 5, 6: Completely healed intra-oral ulcer, chest ulcers and rashes after the medical treatment, 3 months later

\section{Case Report}

A 35-year old man presented to the Oral and Maxillofacial Unit, Teaching Hospital Karapitiya with the chief complaint of multiple oral ulcers with intermittent fever. This was associated with night sweating, anorexia and weight loss of about $15 \mathrm{~kg}$. The patient did not complaint of cough or expectoration. The patient had attacks of fever once or twice a day without chills and there was no arthralgia or myalgia.

His remaining medical history was unremarkable and he denied any sexual misconduct. He was a farmer who occasionally involved in Chena cultivation and used to sleep in the rock cave which has been inhabited by bats. The patient was a nonsmoker but consumed alcohol occasionally. He also had chewed betel quids with all four ingredients for more than ten years.

On admission, there was an ulcerative growth at right upper alveolus involving palate and buccal sulcus, $4 \mathrm{~cm} \times 3 \mathrm{~cm}$ in size with everted margins (Figure 1). In addition to that, he had chronic ulcers on his chest, legs, penis and there was a generalised skin rash (Figures 2 and 3). Laboratory investigations did not reveal any significant abnormality including HIV 1 and 2 antibodies.
Incisional biopsy from the oral lesion was performed. Histopathology with haematoxylin and eosin stained sections and special stains PAS-D (periodic acid schiff-diastase) and Grocott stain concluded granulomatous inflammation due to histoplasmosis (Figure 4).

Abdominal CT and ultrasonography (USS) revealed hepatosplenomegaly. Colonoscopy showed mucous membrane of the colon severely inflamed and apthoid ulcers scattered throughout the mucous membrane. Biopsy of the colon mucosa demonstrated granulomatous inflammation induced by mycotic infection, consistent with histoplasmosis. The condition was diagnosed as progressive disseminated histoplasmosis.

The patient was started with intravenous amphotericin B for 30 days. Then it was followed by oral itraconazole $200 \mathrm{mg}$ mane for 15 days. After completion of the treatment, patient's appetite improved. All the skin rashes diminished and oral, penis and chest ulcers healed completely (Figure $5,6)$.

The follow up CT scan showed spleen returned to normal size. Upper and lower gastrointestinal endoscopy performed 2 months after the treatment revealed healing ulcers in the mucous membranes. 


\section{Discussion}

Histoplasmosis is caused by a dimorphic fungus known as Histoplasma capsulatum that lives in acidic, damp environments and caves where bats or birds reside (2). This dimorphic fungus remainsin mycelia form in the ambient temperature and grows as yeast form in the human lungs. The severity of the disease depends on the quantity of the spores inhaled and the immune state of the patient (3). Disease intensity is influenced by the interaction between innate / adaptive immunity and fungal virulence factors (4).

Four clinical sub types have been described in the literature: acute pulmonary, chronic pulmonary, disseminated and African type. Disseminated form is characterised by progression of the disease to extra pulmonary siteswhich is predominantly seen in the immunocompromised patients such as HIV positive individuals, patients with hematolymphoid malignancies, solid organ transplants and patients who received chemotherapeutic and immunosuppressive agents (5). It can also be developed in 1 case per 2000 cases in adults who are immunocompetent (6).

The disseminated histoplasmosis has different extrapulmonary manifestations such as oral cavity, skin, liver, spleen, adrenal glands, lymph nodes, gastrointestinal tract, kidney and central nervous system (3).

Common extra pulmonary presentation of disseminated histoplasmosis is oral cavity lesions. Up to $66 \%$ of the patients who presented with oral manifestation have disseminated disease (3). The tongue, palate and buccal mucosa are more common sites for oral manifestations (3).Cutaneous dissemination is observed in $38 \%$ to $85 \%$ cases (7). The treatment of choice for disseminated histoplasmosis is intravenous amphotericin B but still there is $7 \%$ to $23 \%$ of mortality rate (3). Azole antifungal agents such as itraconazole also can be used as alternative to amphotericin B or alternate maintenance therapy.

\section{Conclusions}

Disseminated histoplasmosis rarely occurs in an immunocompetent patient as in this case. The oral presentation is easily misdiagnosed for a neoplasm due to the nature of the ulcer and the rarity. Therefore, it is a challenge to make the correct diagnosis and provide proper treatment.

\section{References}

1. Kauffman CA. Histoplasmosis. Clin Chest Med. Jun 2009; 30(2): 217-25.

2. Iqbal F, Schifter M, Coleman HG. Oral presentation of histoplasmosis in an immunocompetent patient: a diagnostic challenge. Australian Dental Journal 2014; 59: 386-8.

3. Rodolfo N Epifanio, Robert B Brannon, Brian C Muzyka. Disseminated histoplasmosis with oral manifestation: Special Care in Dentistry Nov 2007; 27(6): 236-9.

4. Isadora Peres Klein, Marco Antonio Trevizani Martins, Manoela Domingues Martins, Vinicius Coelho Carrard. Diagnosis of HIV infection on the basis of histoplasmosisrelated oral ulceration. Special Care in Dentistry 2016: 36(2): 99-103.

5. Manoj Harnalikar, Vidya Kharkar, Udyakhopkar. Disseminated cutaneous Histoplasmosis in immunocompetent adult. Indian Journal Dermatology 2012; 57(3): 206-9.

6. Cuellar-Rodriguez J, Avery RK, Lard M, Budev M, Gordan SM, Shrestha NK, et al. Histoplasmosis in solid organ transplant recipients: 10 years of experience at a large transplant center in an endemic area. Clin Infect Dis 2009; 49(5): 710-6.

7. Tobon AM, Agudelo CA, Rosero DS, et al. Disseminated histoplasmosis: a comparative study between patients with acquired immunodeficiency syndrome and non-human immunodeficiency virus-infected individuals. Am J Trop Med Hyg 2005; 73: 576-82. 